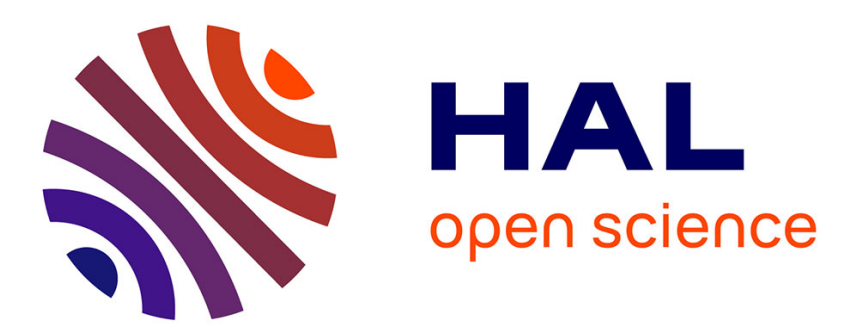

\title{
Les choix politiques des jeunes à l'épreuve du temps : une enquête longitudinale \\ Anne Muxel
}

\section{To cite this version:}

Anne Muxel. Les choix politiques des jeunes à l'épreuve du temps: une enquête longitudinale. Revue Française de Science Politique, 2001, 51 (3), pp.409 - 430. 10.3406/rfsp.2001.403637. hal$01018067 \mathrm{v} 2$

\section{HAL Id: hal-01018067 \\ https://hal-sciencespo.archives-ouvertes.fr/hal-01018067v2}

Submitted on 3 Jul 2018

HAL is a multi-disciplinary open access archive for the deposit and dissemination of scientific research documents, whether they are published or not. The documents may come from teaching and research institutions in France or abroad, or from public or private research centers.
L'archive ouverte pluridisciplinaire HAL, est destinée au dépôt et à la diffusion de documents scientifiques de niveau recherche, publiés ou non, émanant des établissements d'enseignement et de recherche français ou étrangers, des laboratoires publics ou privés. 


\section{Les choix politiques des jeunes à l'épreuve du temps. Une enquête longitudinale}

In: Revue française de science politique, 51e année, n³, 2001. pp. 409-430.

Citer ce document / Cite this document :

Muxel Anne. Les choix politiques des jeunes à l'épreuve du temps. Une enquête longitudinale. In: Revue française de science politique, 51e année, $n^{\circ} 3,2001$. pp. 409-430.

doi : $10.3406 /$ rfsp.2001.403637

http://www.persee.fr/web/revues/home/prescript/article/rfsp_0035-2950_2001_num_51_3_403637 


\section{Abstract}

The stability of [french] youth political choices. A longitudinal survey

Youth is an intensive period of transaction during which individuals, in different -ways, enter adulthood and discover politics. Longitudinal studies allow us to observe not only the different ways of constructing one's «political self», but also the stability or instability of political choices during this relevant period. Some results from a longitudinal survey conducted for twelve years, between 1986 and 1998, on young people aged 18 in 1986, are presented. The cohort was questioned seven times, following the electoral agenda in France during that period (1986, 1988, 1989,1992, 1993,1995 and 1997). The data show significant variations in the sta-bility of political choices throughout the period, depending on whether the measure refers to left-right self-placement, partisan identification or voting. Left-right identification is still the most structured and stable dimension in France, more so than partisan identification, and obviously much more than voting choice. The data also indicate the lasting impact of personal participation in collective action on later political attitudes and behavior.

\section{Résumé}

Les années de jeunesse sont un temps de négociation et d'expérimentation, donc une période privilégiée pour observer le mouvement de formation et d'expression des choix politiques des individus. Seules les études longitudinales et l'observation de panel sur une longue durée per-mettent d'identifier le travail propre du temps sur la construction des choix et des comporte-ments politiques et d'en apprécier la durabilité. Cet article présente des résultats tirés d'une enquête longitudinale menée auprès d'une cohorte de jeunes français âgés au départ de 18 ans, interrogés lors de toutes les grandes élections nationales, pendant près de douze ans c'est-à-dire jusqu'à ce qu'ils atteignent l'âge de 30 ans. Sept vagues d'interrogations successives (1986, 1988, 1989, 1992, 1993, 1995,et 1997) ont été conduites. Les résultats de l'étude révè-lent une structuration à la fois persistante et stable du clivage gauche-droite et corrélativement une plus grande fragilité du lien partisan ainsi qu'une volatilité encore plus marquée des choix électoraux. Ils montrent l'empreinte de la participation à une expérience politique collective sur les attitudes et les comportements politiques ultérieurs.

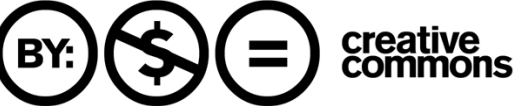




\title{
LES CHOIX POLITIQUES DES JEUNES À L'ÉPREUVE DU TEMPS UNE ENQUÊTE LONGITUDINALE
}

\author{
ANNE MUXEL
}

$\mathrm{L}$

es années de jeunesse délimitent, par leurs caractéristiques mêmes, une période d'observation particulièrement pertinente pour étudier la socialisation politique. Elles définissent un espace de transition entre la socialisation primaire, caractérisée par une situation d'hétéronomie propre au statut d'enfant, et les phases ultérieures façonnant la socialisation secondaire de l'individu conduisant progressivement à l'affirmation d'une autonomie, tant en termes de statut que de légitimité des choix. C'est donc un temps de passage, au travers duquel se réalise un changement d'état, résultant d'une totalisation complexe des déterminismes comme des initiatives multiples orientant l'action des individus et façonnant leurs systèmes de valeurs et d'opinions '.

Interroger les formes du lien en politique dans ce temps spécifique de la vie, c'est donc affirmer bien plus qu'un parti pris méthodologique, c'est fixer un cadre d'analyse correspondant à une conception interactionniste et dynamique de la socialisation politique des individus. Il s'agit de l'étudier comme un processus, résultant d'opérations, simultanées ou successives, de construction, de formalisation, d'interprétation et enfin d'application, de l'ensemble des codes, des représentations et des pratiques, au travers desquels l'individu appréhende l'univers politique, s'y oriente et y adopte des comportements ; cela au fil du temps et des étapes qui marquent son parcours de vie.

Pour P. Berger et Th. Luckman, la socialisation suppose une « prise en charge » par l'individu lui-même de ce processus, qui se fait au travers d'un « équilibrage continuel » entre intériorité et extériorité. Ils définissent la socialisation comme « l'installation consistante et étendue d'un individu à l'intérieur du monde objectif d'une société ou d'un secteur de celle-ci » ${ }^{2}$. Cette installation requiert des processus d'ajustement de temporalités différenciées, ne s'agençant pas nécessairement dans un calendrier linéaire ou cohérent, au travers desquels l'arbitrage individuel des choix politiques pourra s'effectuer. Il faut donc réinscrire l'expérience du sujet dans une durée, dans un parcours. En bout de course, comprendre la socialisation c'est pouvoir tracer une trajectoire, évaluer une distance parcourue, saisir les enchaînements de circonstances, repérer les seuils, les points de passage, les moments de cristallisation.

Le temps même est un opérateur efficient dans ce travail de totalisation de l'identité du sujet. Il oblige à une constante réévaluation des modalités comme des contenus

1. Sur cette problématique du « moratoire psycho-social » propre aux années de jeunesse, on peut se reporter à E. E. H. Erikson, Adolescence et crise. La quête de l'identité, Paris, Flammarion, 1972. Sur les caractéristiques d'un moratoire en politique, on peut consulter le chapitre 4 de mon ouvrage, L'expérience politique des jeunes, Paris, Presses de Sciences Po, 2001.

2. Peter Berger, Theodor Luckman, La construction sociale de la réalité, Paris, Méridiens-Klincksiek, 1986 (pour la traduction française), p. 179. Plus spécifiquement sur la socialisation politique, on peut se référer à l'ouvrage d'Annick Percheron, La socialisation politique, Paris, Armand Colin, 1993. 


\section{Anne Muxel}

de la socialisation, au gré des étapes de l'existence, dans l'entremêlement des temporalités individuelles et des temporalités sociales, historiques et politiques. Comprendre le processus de construction de l'identité politique des individus suppose aussi que soient démêlés les effets entrecroisés de l'âge, de la génération et de la période '. C'est dans cet écheveau tissé de déterminismes - réels et symboliques, individuels et collectifs -, et d'innovations - plus ou moins profondes, plus ou moins en rupture avec les générations précédentes -, que se dessine une trajectoire politique, faite de choix, de représentations et de pratiques.

Seules les études longitudinales et l'observation de panel sur une longue durée permettent d'identifier le travail propre du temps sur la construction des choix et des comportements politiques et d'en apprécier la durabilité. Mais si leur intérêt n'est plus à démontrer, elles restent rares, car difficiles à réaliser ${ }^{2}$.

En France, l'observation sur une longue durée des interférences entre la socialisation familiale, les conditions de l'insertion sociale et professionnelle, et la mise en place des attitudes et des comportements politiques, n'a pratiquement jamais été menée. C'est à cette lacune que la recherche que j'ai mise en place, il y a maintenant une douzaine d'années tente de répondre. L'approche longitudinale permet de développer une démarche compréhensive s'attachant à situer l'individu dans un temps à la fois continu et discontinu, car le temps historique comme le temps biographique ne sont ni linéaires ni homogènes. Et surtout, elle invite à privilégier la question du changement, c'est-à-dire la question de la transformation, du passage d'un état à un autre, les mouvements et les déplacements qui formeront au bout du compte le tracé de la trajectoire politique d'un individu.

1. Karl Mannheim, Le problème des générations, Paris, Nathan, 1990 (pour la traduction française).

2. Travailler sur la socialisation politique nécessite souvent des moyens lourds et des dispositifs d'enquête s'inscrivant dans une durée longue, autant de contraintes qui ne sont pas propices à l'engagement des chercheurs. Cette tendance est commune à toute la communauté scientifique internationale et nationale. La France n'est pas seule dans ce cas. La recherche américaine, une figure de proue pourtant des études sur la socialisation politique dans les années 1960 et 1970, n'a plus pendant longtemps fourni de travaux novateurs. Depuis le dernier ouvrage important de Kent Jennings et de Richard Niemi, Generations and Politics, Princeton, Princeton University Press, 1981, qui, d'une certaine façon, par l'ampleur de l'observation menée ainsi que par la richesse des résultats fournis, était une sorte d'aboutissement de la recherche dans le champ de la socialisation politique, peu de recherches d'une telle ampleur ont été menées. En Europe, le constat est identique, même si des recherches récentes menées dans les pays européens témoignent d'un regain d'intérêt pour le sujet, mais elles restent relativement dispersées et isolées. En Grande-Bretagne, si l'étude de l'équipe de Hilde Himmelweit, How Voters Decide. A Longitudinal Study of Political Attitudes and Voting Extending over Fifteen Years, Londres, Academic Press, 1981, reste une référence en la matière mais date un peu, d'autres enquêtes longitudinales ont vu le jour récemment, dont celle conduite par J. Bynner, E. Ferri et P. Sheperd, Twenty-Something in the 1990s. Getting on, Getting by, Getting Nowhere, Aldershot, Asgate Publishing, 1997, qui ont suivi une même cohorte de jeunes, nés la même semaine en 1970, depuis leur naissance jusqu'au début des années 1990. Dans les pays d'Europe du Sud, en Italie et en Espagne, certaines équipes de recherche ont entamé récemment des enquêtes sur la socialisation politique des jeunes générations. On peut se reporter notamment à l'ouvrage collectif dirigé par Gianfranco Bettin, Giovani e democrazia in Europa, Milan, Cedam, 1999. En France, on doit à Vincent Tournier d'avoir réalisé une enquête récente sur un échantillon apparié de parents et d'enfants mettant au jour des résultats intéressants, notamment en ce qui concerne l'imbrication des rôles de la famille et de l'école sur l'apprentissage politique. Cf. son article «École publique, école privée, le clivage oublié », Revue française de science politique, 47 (5), octobre 1997. 


\section{LE PANEL : UN OUTIL POUR DÉROULER LE TEMPS}

Le temps représente sans aucun doute la dimension la plus décisive pour comprendre les ressorts de la construction de l'identité politique d'un individu, mais aussi ce qu'il y a de plus difficile à saisir.

J'ai réalisé une enquête longitudinale pour suivre les conditions d'entrée en politique d'une même génération de jeunes français âgés de 18 ans, en les interrogeant lors de toutes les grandes élections nationales, pendant près de douze ans, c'est-à-dire jusqu'à ce qu'ils atteignent l'âge de 30 ans. J'ai conduit sept vagues d'interrogations successives $(1986,1988,1989,1992,1993,1995$,et 1997) '. Partie avec 3508 jeunes, l'enquête prend fin douze ans plus tard en en comptant encore 603 dans son échantillon final, approchant tous la trentaine aujourd'hui.

Le rythme de l'interrogation du panel suit la périodisation de la vie politique française depuis une dizaine d'années. Première interrogation au moment du mouvement lycéen-étudiant de novembre-décembre 1986, dernière interrogation au lendemain des élections législatives de 1997. Entre les deux, le second septennat de François Mitterrand, les deux périodes de cohabitation, 1986-1988 et 1993-1995, l'élection de Jacques Chirac en 1995, la dissolution de l'Assemblée nationale et les élections législatives anticipées en 1997. Cela pour la vie politique nationale. Mais c'est aussi la période de la réalisation de l'Union européenne (élections européennes en 1989 et en 1994, référendum pour la ratification du traité de Maastricht en 1992), de la chute du Mur de Berlin en 1989, de la guerre en ex-Yougoslavie. Ce sont les grandes dates, les grands repères et le calendrier d'une sorte de carnet de bord événementiel et conjoncturel de la période politique dans laquelle ces années d'apprentissage se situent.

1. Sept interrogations ont été réalisées :

- Fin décembre 1986-janvier 1987: 4334 jeunes âgés de 17 à 19 ans ont été interrogés à l'aide d'un questionnaire auto-administré distribué dans les établissements scolaires dans lesquels les jeunes étaient scolarisés, soit dans des foyers de jeunes travailleurs, soit enfin au travers de l'ANPE. Il s'agit d'un échantillon diversifié et large, composé de jeunes scolarisés dans des lycées d'enseignement général, d'enseignement technique et d'enseignement professionnel de la Région parisienne, de jeunes scolarisés dans les quatre écoles de métiers EDF-GDF en France, et de jeunes ayant quitté le système scolaire, salariés ou chômeurs.

À partir de cet échantillon initial de 4334 jeunes, un panel a été constitué portant le nombre d'individus à 3508 .

- Mai 1988 : réinterrogation du panel au lendemain du $2^{c}$ tour de l'élection présidentielle. Sur les 3508 questionnaires envoyés par courrier, 2378 ont été retournés (taux de réponse : $68 \%)$.

- Mars 1989 : troisième interrogation du panel au lendemain du $2^{c}$ tour des élections municipales. Sur les 2378 questionnaires envoyés, 1959 ont été retournés (taux de réponse : $82 \%)$.

- Mars 1992 : quatrième interrogation du panel au lendemain des élections régionales du 22 mars. Sur les 1959 questionnaires envoyés, environ 1080 ont été retournés (taux de réponse : $55 \%$ ).

- Mars 1993 : cinquième interrogation du panel au lendemain du deuxième tour des élections législatives, 1080 questionnaires ont été envoyés, 960 ont été retournés (taux de réponse : $88 \%$ ).

- Mai 1995 : sixième interrogation du panel au lendemain du deuxième tour de l'élection présidentielle, 960 questionnaires ont été envoyés, 733 ont été retournés (taux de réponse : $76 \%$ ).

- Mai 1997 : septième et dernière interrogation au lendemain du deuxième tour des élections législatives, 733 questionnaires ont été envoyés, 603 questionnaires ont été retournés (taux de réponse : $82 \%$ ), qui constituent l'échantillon final, sur lequel porte l'analyse. 
Anne Muxel

Tableau 1. Sept vagues d'enquête

\begin{tabular}{ccccccc}
\hline Vague 1 & Vague 2 & Vague 3 & Vague 4 & Vague 5 & Vague 6 & Vague 7 \\
1986 & 1988 & 1989 & 1992 & 1993 & 1995 & 1997 \\
nov.-déc. & Prés. & Mun. & Rég. & Lég. & Prés. & Lég. \\
\hline 18 ans & 20 ans & 21 ans & 24 ans & 25 ans & 27 ans & 29 ans \\
$(\mathrm{N}=3508)$ & $(\mathrm{N}=2378)$ & $(\mathrm{N}=1982)$ & $(\mathrm{N}=1190)$ & $(\mathrm{N}=960)$ & $(\mathrm{N}=764)$ & $(\mathrm{N}=603)$ \\
\hline
\end{tabular}

À ce cadrage temporel il faut ajouter des indicateurs d'appréciation et des critères d'évaluation plus qualitatifs, moins facilement objectivables, mais néanmoins pertinents pour caractériser ces dix dernières années en France : crise de la représentation politique et de l'adhésion, recomposition des appartenances, effritement du lien partisan, perception négative de la politique, brouillage des repères idéologiques, introduction de nouvelles lignes de clivages politiques et culturels, prédominance des problématiques économiques, mondialisation de l'économie, élargissement des territoires d'appartenance, persistance et aggravation de la crise sociale et économique ${ }^{1}$.

La possibilité d'observer des individus sur une durée aussi longue permet d'analyser ce qui est généralement laissé dans l'ombre et l'opacité, faute de données : les raisons de la constance ou de l'instabilité dans les attitudes et les comportements politiques, le processus de formation des choix, le devenir du premier vote, l'entremêlement des calendriers socio-biographiques, socio-professionnels et politiques.

Dans le cadre de cet article, je tenterai une mesure des effets du temps sur le parcours politique d'un individu en fonction de deux axes problématiques. Le premier renvoie à la durabilité des positionnements et la mesure effectuée mettra en évidence l'existence d'une stabilité différenciée des choix politiques selon qu'ils sont appréhendés au travers de l'autoclassement sur une échelle gauche-droite en sept positions, ou au travers de la proximité partisane, ou encore au travers des votes. Le second s'attache aux effets d'imprégnation et de cristallisation des orientations et des comportements politiques. II s'agira d'apprécier l'empreinte d'une expérience politique collective et d'une participation effective sur les choix ultérieurs de l'individu et de mettre au jour l'existence de moments forts de cristallisation au cours desquels le temps biographique et le temps historique s'articulent et se composent pour créer des effets durables.

Mais avant de montrer les effets du temps sur la structuration de l'identité politique des individus, il faut évoquer les effets du temps sur le panel lui-même et donc les difficultés méthodologiques auxquelles se heurtent les études longitudinales. Les deux principaux obstacles sur lesquels butte généralement ce type d'analyse sont la fidélisation du panel au cours du temps, d'une part, la distorsion de l'échantillon du fait de son irrémédiable réduction, d'autre part.

\section{UNE ÉVALUATION DES RISQUES DE FIDÉLISATION DU PANEL}

Pour apprécier les risques de fidélisation du panel, la mesure de l'évolution de l'intérêt pour la politique tout au long des șept vagues d'enquête peut servir de test.

1. On peut notamment se reporter à l'article de Pascal Perrineau «Le premier tour des élections législatives de 1997 », Revue française de science politique, 47 (3-4), juin-août 1997, ou encore à l'ouvrage collectif dirigé par Daniel Boy et Nonna Mayer, L'électeur a ses raisons, Paris, Presses de Sciences Po, 1997. 


\section{À l'épreuve du temps}

En effet, le fait d'interroger les enquêtés à sept reprises sur leurs attitudes et sur leurs comportements politiques pourrait modifier à termes leur rapport à la politique, notamment en accroissant leur sensibilité en la matière et en développant progressivement leur intérêt. Or il n'en est rien. Excepté l'écart de 39 points constaté entre les vagues 1 et 2 , vraisemblablement lié au fait que la mortalité du panel fut proportionnellement plus élevée au début de l'enquête que dans son cours ultérieur, les différences du niveau d'intérêt pour la politique ne permettent pas de conclure à un effet de fidélisation du panel (tableau 2). Si tel avait été le cas, le niveau d'intérêt aurait dû augmenter de façon linéaire et régulière. Il n'y a donc pas d'effet de fidélisation. Les variations observées sont liées aux effets du contexte et de la conjoncture politiques. Les deux pics d'intérêt maximum coincident avec les élections présidentielles de 1988 et de 1995. Les élections présidentielles sont toujours un moment de plus forte sollicitation politique et enregistrent toujours un taux de participation électorale plus conséquent que pour tout autre type d'élection. Les mouvements observés quant à l'intérêt pour la politique traduisent cet effet de contexte.

Tableau 2. Fidélisation du panel mesurée au travers de l'intérêt pour la politique

\begin{tabular}{cccccccc}
\hline & Vague 1 & Vague 2 & Vague 3 & Vague 4 & Vague 5 & Vague 6 & Vague 7 \\
& 1986 & 1988 & 1989 & 1992 & 1993 & 1995 & 1997 \\
\hline & 18 ans & 20 ans & 21 ans & 24 ans & 25 ans & 27 ans & 29 ans \\
Intérêt ++ et + & $35 \%$ & $74 \%$ & $59 \%$ & $49 \%$ & $51 \%$ & $67 \%$ & $60 \%$ \\
\hline
\end{tabular}

Lazarsfeld avait lui-même montré lors de l'un des premiers panels politiques réalisé aux États-Unis, à Elmira, la quasi-absence de tout biais de conditionnement sur les questions politiques '. Ayant réalisé des entretiens répétés à plusieurs reprises dans le temps, il avait dû admettre que cette répétition n'avait pas modifié les résultats de l'enquête, qu'elle avait tout au plus eu une incidence sur la diminution des sans réponse.

\section{LES EFFETS DU TEMPS SUR LA STRUCTURE DE L'ÉCHANTILLON}

Mais l'une des questions les plus dérangeantes, et certes problématique, est la mortalité du panel, sa réduction au fil des années. $17 \%$ de 1'échantillon initial, c'està-dire des enquêtés présents lors de la première vague d'enquête en 1986, ont pu être suivis jusqu'au bout, $25 \%$ si l'on mesure la déperdition par rapport à 1988.

Lorsque l'on examine les taux de mortalité du panel, on s'aperçoit que celui-ci est d'autant plus fort que l'intervalle de temps entre deux interrogations est grand (tableau 3). À l'exception de la dernière vague où se mesure un effet de la stabilisation de la cohorte, et peut être aussi un début de fidélisation. À cela il faut ajouter les effets de l'âge lui-même qui scande des césures sans doute importantes dans cette période de la vie (c'est entre les vagues 3 et 4, c'est-à-dire entre les âges de 21 et 24 ans que l'attrition est la plus forte).

1. Paul Lazarsfeld, The People's Choice. How Voter Makes up his Mind in a Presidential Campaign, New York, Columbia University Press, 1948 [1 $1^{\text {rc }}$ éd. : 1944]. 
Tableau 3. Taux de mortalité du panel

\begin{tabular}{cccccc}
\hline$V 1 \rightarrow V 2$ & $V 2 \rightarrow V 3$ & $V 3 \rightarrow V 4$ & $V 4 \rightarrow V 5$ & $V 5 \rightarrow V 6$ & $V 6 \rightarrow V 7$ \\
$1986-1988$ & $1988-1989$ & $1989-1992$ & $1992-1993$ & $1993-1995$ & $1995-1997$ \\
\hline $32 \%$ & $18 \%$ & $45 \%$ & $12 \%$ & $21 \%$ & $21 \%$ \\
\hline
\end{tabular}

Malgré l'incontestable pesanteur de cette mortalité, il faut reconnaître $a$ contrario, que le pari de maintenir sur le long terme un échantillon d'une taille suffisante est par ailleurs réussi. En 1986, j'avais en tête que, lorsqu'il n'en resterait que $500, j$ 'arrêterai, et j'avais alors fixé une borne d'âge qui me semblait lointaine, 25 ans, soit sept ans plus tard. J'ai pu maintenir le panel cinq années supplémentaires, reculant le temps d'observation des enquêtés jusqu'à l'âge de la trentaine. Et j'aurais même pu poursuivre encore, puisque la dernière vague en comptabilise 6031 .

Mais si l'érosion de l'effectif global de l'échantillon a été contrôlée de façon relativement satisfaisante, le problème de la différenciation non seulement sociale mais aussi politique de cette mortalité reste entier. En effet, les individus ayant répondu à l'ensemble des sept vagues présentent-ils les mêmes caractéristiques que les individus qui ont été perdus en cours de route? Il faudrait pouvoir conduire un travail méthodologique spécifique sur cette question, en essayant de retrouver ceux qui ont cessé de répondre et en les réinterrogeant. Toutefois à partir des données existantes, les effets de cette mortalité différenciée apparaissent relativement maîtrisés, en tout cas d'un point de vue structurel.

Tableau 4. Évolution de la structure de l'échantillon selon le sexe et l'origine sociale des enquêtes (\%)

\begin{tabular}{lcc}
\hline & $\begin{array}{c}1986 \\
\text { Vague } 1 \\
(n=3508) \\
18 \text { ans }\end{array}$ & $\begin{array}{c}1997 \\
\text { Vague } 7 \\
(n=603) \\
29 \text { ans }\end{array}$ \\
\hline - Filles & 42 & 46 \\
- Garçons & 58 & 54 \\
\hline CSP du père : & & \\
- Indépendants & 14 & 14 \\
- Cadres et professions intellectuelles supérieures & 21 & 27 \\
- Professions intermédiaires & 20 & 23 \\
- Employés & 13 & 11 \\
- Ouvriers & 20 & 20 \\
- Sans réponse & 12 & 5 \\
\hline
\end{tabular}

1. Ces taux de réponse, très satisfaisants pour une enquête auto-administrée s'étant déroulée par voie postale, ont été obtenus grâce à une gestion rigoureuse du fichier d'adresses, à une recherche active des individus ayant déménagé d'une vague à l'autre, à partir notamment de l'adresse des parents que nous avions pris soin de conserver, grâce à des relances systématiques par téléphone et par courrier à chaque phase de l'enquête, enfin et surtout grâce à un contact qui a toujours été maintenu avec les enquêtés. J'ai envoyé aux jeunes des informations sur l'état d'avancement de l'enquête, un effort qui a pu assurer le succès de l'enquête en les engageant à répondre à chaque nouveau questionnaire. 


\section{À l'épreuve du temps}

La structure socio-démographique de l'échantillon ne subit pas de distorsions importantes au fil du temps. En effet, l'origine sociale, appréhendée par la catégorie socio-professionnelle du père, se retrouve assez également distribuée entre la vague 1 et la vague 7 . Par ailleurs, le déséquilibre dans la répartition du nombre des filles et des garçons de l'échantillon initial, bien que se corrigeant quelque peu au fil du temps, persiste (tableau 4).

Mais pour bien comprendre les risques de biais engendrés par la mortalité du panel, il faut aussi raisonner en termes de trajectoires individuelles. Car s'il n'y a pas de réelle différenciation de la structure, en revanche, il y en a du point de vue de la trajectoire scolaire et professionnelle des individus eux-mêmes. Reprenons les 603 enquêtés qui composent le panel, soit les individus qui ont répondu à la totalité des sept vagues, et comparons leurs trajectoires dans le temps par rapport aux caractéristiques de l'échantillon d'origine, lors de la vague 1 (tableau 5).

Tableau 5. Évolution de la structure de l'échantillon selon le parcours scolaire et professionnel des enquêtés (\% en colonne)

\begin{tabular}{lccc}
\hline & $\begin{array}{c}1986 \\
\text { Vague } 1 \\
(n=3508)\end{array}$ & $\begin{array}{c}1992 \\
\text { Vague } 4 \\
(n=1190)\end{array}$ & $\begin{array}{c}1997 \\
\text { Vague } 7 \\
(n=603)\end{array}$ \\
\hline Niveau de diplôme & 18 ans & 24 ans & 29 ans \\
- Sans diplôme & 12 & - & - \\
- CAP-BEP & 21 & 13 & 11 \\
- Bac général/technique & 67 & 27 & 16 \\
- DEUG-BTS & - & - & 26 \\
- Université + 3 ans & - & 16 & 27 \\
- Grandes Écoles & - & 2 & 13 \\
- Autres & - & 8 & 7 \\
\hline Situation professionnelle & - & 42 & 14 \\
- Contrat CDD & - & 2 & 62 \\
- Contrat CDI & - & 4 & 6 \\
- Emploi précaire & - & 4 & 4 \\
- Chômeurs & - & 26 & 3 \\
- Étudiants & - & 14 & 5 \\
- Étudiants salariés & - & 8 & 6 \\
- Autres & & &
\end{tabular}

Qu'observe-t-on? Tout d'abord la réduction du nombre d'étudiants au fil du temps et au fur et à mesure de l'entrée progressive du panel dans la vie active. Le moment de passage décisif entre le temps des études et le temps d'entrée sur le marché du travail se fait entre les vagues 3 et 4 , soit entre 21 et 24 ans. À partir de la vague 4 les actifs sont toujours majoritaires.

On constate une réelle mortalité différenciée du panel selon le niveau de formation initial des enquêtés. Le niveau de diplôme a joué un rôle décisif dans la diminution de l'échantillon au cours des trois premières vagues d'enquête, c'est-à-dire dans les toutes premières années du temps de la jeunesse. L'absence de diplôme ou une faible qualification sont des variables très clivantes du point de vue des attitudes et des comportements politiques. Et ce profil d'individus a disparu plus tôt et plus rapidement de l'échantillon, étant donné le contenu de l'enquête et ses modalités de passa- 


\section{Anne Muxel}

tion. Toutefois, il reste en bout de parcours, lors de la vague 7 , une proportion non négligeable de jeunes faiblement diplômés. Quatre profils d'insertion sociale, ont été reconstitués à partir de la trajectoire scolaire et professionnelle des individus sur l'ensemble de la période d'observation : $24 \%$ des enquêtés se sont déclarés étudiants au moins 5 fois sur les 7 vagues d'enquête (passage à la vie active à la vague 6 ou à la vague 7 , soit après 25 ans), $16 \%$ se sont déclarés étudiants 4 fois (passage à la vie active à la vague 5, soit aux alentours de 23-24 ans), $37 \% 3$ fois (passage à la vie active à la vague 4 , soit à $22-23$ ans), $24 \%$ moins de 3 fois, c'est-à-dire qu'ils ont suivi une filière d'études courte et sont pas ou faiblement diplômés.

Il reste à apprécier la façon dont l'échantillon a été affecté en fonction des attitudes politiques des individus, à en saisir l'incidence sur la déperdition du panel. Le terrain des opinions est nécessairement mouvant. Si un individu ne peut pas changer d'origine sociale au cours du temps, en revanche, il peut changer d'opinion, ce qui pour l'analyse complique un peu les choses. S'ajoute à cela le fait que ces changements d'opinions sont mesurés à partir d'intervalles de temps non réguliers, ce qui d'un point de vue strictement statistique rend l'analyse longitudinale des données relativement difficile. Mais si l'on retient l'indicateur de classement sur une échelle gauche-droite en sept positions, on peut constater une mobilité relative des classements au fil du temps, du point de vue des individus, mais peu de distorsions, là encore, du point de vue de la structure de l'échantillon.

\section{LES EFFETS DU TEMPS SUR LA STRUCTURATION DE L'IDENTITÉ POLITIQUE}

L'intérêt d'un panel est de permettre de comprendre comment s'agencent les différents calendriers d'entrée en politique, d'en suivre au plus près l'agenda. La diversité des temporalités mises au jour, ainsi que les profils d'élaboration des choix, s'expliquent en partie par les effets conjugués des processus d'identification et des processus d'expérimentation. Le temps de la jeunesse, parce qu'il est le temps par excellence de l'insertion dans la vie sociale, de la construction d'une identité politique personnelle, met sans doute davantage qu'à d'autres moments de la vie la politique à l'épreuve de la réalité. En ce sens, il est un temps moratoire, régi par l'expérimentation. Mais expérimentation ne veut pas dire que les facteurs à l'œuvre dans la transmission familiale, ou encore que l'ensemble des dispositions acquises au cours de la socialisation primaire, n'ont plus d'emprise. L'expérimentation selon le type de filiation ne désigne pas une même logique d'intégration politique '.

$L$ 'analyse longitudinale peut restituer le mouvement à l'œuvre, en figurer les circonvolutions et les amplitudes, selon les moments et les circonstances du parcours effectué, mais aussi selon les dimensions et les opérateurs de l'univers politique par rapport auquel on veut l'évaluer. La trame ainsi représentée fait apparaître l'entrelacs de la continuité et de la discontinuité, mais aussi de la constance et de la mobilité, dans la formation et dans l'expression des choix.

1. Pour une explicitation des différents types de trajets d'entrée en politique, on peut se reporter à mon article «L'âge des choix politiques. Une enquête longitudinale auprès des $18-$ 25 ans », Revue française de sociologie, 33, 1992. 


\section{LA RELATIVE STABILITÉ DU CLIVAGE GAUCHE-DROITE}

Entre les vagues 1 et $7,18 \%$ des individus sont restés strictement fidèles au même classement sur l'échelle gauche-droite, en se classant à sept reprises sur la même case. Une majorité d'individus, bien que revendiquant une orientation à gauche ou à đroite, a bougé à l'intérieur de chacun de ces deux camps : $25 \%$ se sont montrés mobiles à l'intérieur des positions de gauche $(1,2$ ou 3$)$ et $28 \%$ à l'intérieur des positions de droite (5,6 ou 7). Selon le choix de la grille de lecture que l'on adoptera, ils pourront être considérés comme des «mobiles » intra-gauche ou intradroite, ou bien comme des «stables » intra-gauche ou intra-droite, si l'on privilégie leur fidélité au camp initialement choisi. Mais ils sont en tout point différents des vrais mobiles ou vrais instables $(11 \%)$, qui, eux, ont franchi au moins une fois au cours de la période d'observation, la barrière gauche-droite. Restent les indéterminés, $18 \%$ de l'échantillon, qui ont refusé de répondre ou qui ont choisi de se positionner sur la case centrale de l'échelle au moins quatre fois sur les sept que compte la période d'observation.

Si la constance des positionnements en termes de gauche et de droite domine largement, elle doit compter avec l'importance de la mobilité interne. Il faut donc penser le mouvement à l'œuvre dans une dialectique complexe. D'une part, il n'y a pas de constance ni de continuité pures, derrière la permanence, le jeu volatile des recompositions possibles est toujours à l'œuvre. D'autre part, le changement n'est pas non plus monolithique ou univoque, il résulte de micro-déplacements, de translations et de glissements, souvent peu perceptibles. Le mouvement des classements entre les vagues 1 et 7 enregistre une activité relativement importante (tableau 6 ). Les cases formant la diagonale du tableau n'enregistrent jamais un taux de correspondance supérieur à $52 \%$, et l'on y observe des positions potentiellement plus stables que d'autres, la case centrale cristallisant la marge d'incertitude et aussi la plus grande amplitude de mobilité.

Tableau 6. Mouvement des classements sur l'échelle gauche-droite entre la vague 1 et la vague $7(n=603)(\%)$

\begin{tabular}{lcrrrrrrr}
\hline \multicolumn{1}{c}{$V .1 \rightarrow V .7$} & \multicolumn{1}{c}{$l$} & 2 & 3 & 4 & 5 & 6 & 7 & $S R$ \\
\hline $1(\mathrm{n}=7)$ & - & - & - & - & - & - & - & - \\
$2(\mathrm{n}=40)$ & 8 & $\mathbf{5 0}$ & 28 & 8 & 2 & - & - & 4 \\
$3(\mathrm{n}=134)$ & - & 17 & $\mathbf{5 2}$ & 18 & 5 & 2 & 2 & 4 \\
$4(\mathrm{n}=151)$ & 1 & 8 & 30 & $\mathbf{2 8}$ & 25 & & - & - \\
$5(\mathrm{n}=143)$ & - & 2 & 8 & 18 & $\mathbf{5 2}$ & 16 & 1 & 3 \\
$6(\mathrm{n}=45)$ & - & 2 & 4 & 14 & 40 & $\mathbf{3 1}$ & 4 & 5 \\
$7(\mathrm{n}=12)$ & - & - & - & - & - & - & - & - \\
SR $(\mathrm{n}=62)$ & 8 & 8 & 31 & 27 & 18 & 3 & 2 & 3 \\
\hline
\end{tabular}

Lire le tableau ainsi : sur les 134 individus s'étant classés en position 3 lors de la vague $1,17 \%$ se classent en $2,52 \%$ en $3,18 \%$ en $4,5 \%$ en $5,2 \%$ en 6 et $2 \%$ en 7 , lors de la vague 7

Si l'on totalise la part des indécis, définis comme les jeunes s'étant classés à sept reprises sur la case centrale de l'échelle, ou en tout cas qui utilisent au moins trois fois cette possibilité de non-positionnement, $22 \%$ des jeunes se situent en dehors d'un 


\section{Anne Muxel}

choix clairement marqué à gauche ou à droite '. Les autres, soit plus des trois quarts de l'échantillon (78\%), même s'ils y expriment des choix mobiles et fluctuants, se repèrent et se reconnaissent sur cette échelle de partition de l'espace politique. Toutefois l'appréhension de celle-ci varie fortement selon les types de classement opérés et l'on observe une stabilité différenciée selon les positions choisies. L'étude de la fréquence des classements effectués sur chaque case de l'échelle tout au long de la période d'observation fait apparâ̂tre une courbe en $W$, révélant deux pics d'instabilité aux deux extrêmes ( 1 et 7 ) et dans une moindre mesure un pic de même type au centre (4), d'une part, une stabilité plus affirmée sur les positions de centre gauche et de centre droit ( 3 et 5), d'autre part (graphique 1).

Les deux positions extrêmes n'enregistrent aucun ancrage durable. Si près de $10 \%$ des jeunes de l'échantillon s'y sont classés au moins une fois tout au long de la période d'observation, ce choix est resté pour la majorité d'entre eux occasionnel : $50 \%$ de ceux qui se sont classés à l'extrême gauche et $59 \%$ de ceux qui se sont classés à l'extrême droite n'ont fait ce choix qu'une seule fois et ne l'ont pas renouvelé.

Les positions 2 et 6 (respectivement $23 \%$ et $27 \%$ des classements) connaissent une stabilité relative, puisque près du quart des jeunes se situent en $2(24 \%)$, et $29 \%$ de ceux qui se portent en 6 , renouvellent ce même choix au moins quatre fois sur les sept que compte la période d'observation.

Graphique 1. Stabilité au cours des 7 vagues d'enquête de l'autoclassement sur l'échelle gauche-droite en 7 positions

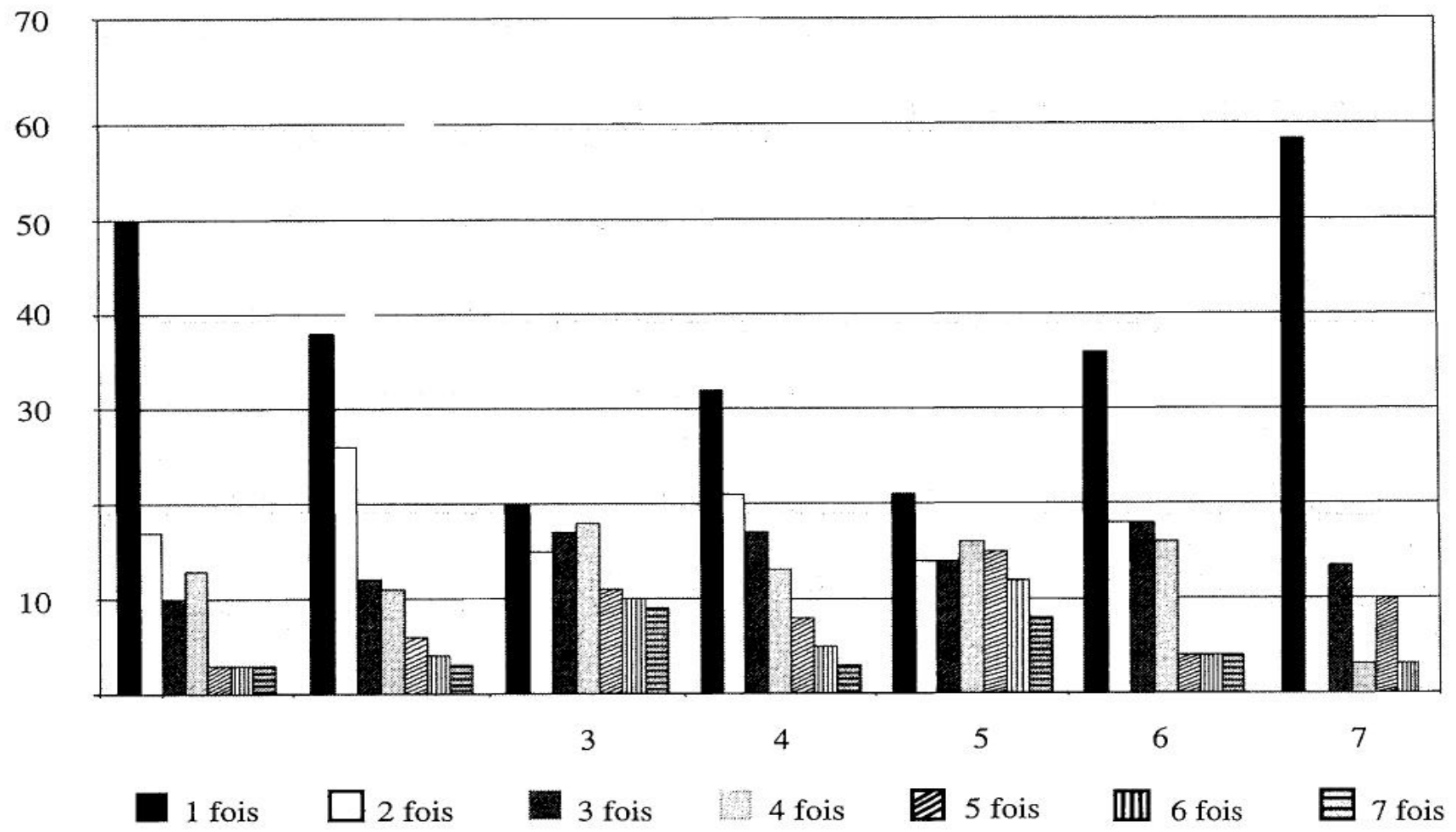

1. Avec un autre mode de calcul, consistant à exclure les jeunes utilisant la case centrale même de façon stable, la stabilité de ceux qui ont non seulement strictement répété le choix d'une même position sur l'échelle, mais d'une position marquée à gauche (1,2 ou 3$)$ ou à droite $(5,6$ ou 7$)$, est ramenée à la proportion de $10 \%$. 
Mais ce sont les positions se rapprochant du centre, 3 et 5 , qui apparaissent à la fois comme les plus utilisées (46\% de l'ensemble des classements) et comme les plus stables. Près de la moitié des jeunes se positionnant en $3(48 \%)$ et plus de la moitié de ceux qui se classent en $5(52 \%)$, réitèrent ce même classement au moins quatre fois. De toutes les positions sur l'échelle, ce sont celles qui suscitent la fidélité la plus forte : $9 \%$ des classements en 3 sont répétés à sept reprises et $8 \%$ des classements en 5 .

Reste la position centrale qui apparaît comme particulièrement volatile et semble utilisée comme un positionnement par défaut et comme un espace de transit. La fréquence et la mobilité qu'elle enregistre en témoignent. $57 \%$ des jeunes utilisent ce classement au moins une fois en onze ans. En revanche, ils sont peu nombreux à renouveler plusieurs fois ce choix. Pour la plupart, il s'agit d'une réponse temporaire, révélatrice des incertitudes et des fluctuations pesant sur les choix partisans ou électoraux : $53 \%$ se classent en 4 seulement une fois ou deux au cours de la période, seuls $16 \%$ réitèrent ce positionnement cinq fois et plus.

Les déplacements sur l'échelle gauche-droite effectués entre les vagues 1 et 7 laissent entrevoir le jeu des affinités et des recompositions à l'intérieur de chaque camp politique, et donc présager des conséquences que celles-ci peuvent avoir notamment lors des élections. Si l'on examine sur la même période la mobilité des choix relatifs à la proximité partisane ou encore au vote, mettant en cause plus directement la question de l'offre politique, les conséquences de cette mobilité intra-gauche et intra-droite sont visibles et révélatrices de la diversité des formes de lien politique, et plus étroitement des formes du lien partisan.

\section{LA FRAGILITÉ DU LIEN PARTISAN}

Interrogés sur les partis dont ils se sentent les plus proches à chaque vague d'enquête ', la consistance des choix des jeunes ne s'établit pas de la même façon sur l'échiquier partisan. Leurs liens, plus ou moins fidèles, plus ou moins lâches, sont assez contrastés.

Tableau 7. Fidélité partisane (\% en colonne)

\begin{tabular}{lccccccc}
\hline $\begin{array}{c}\text { Se sont déclarés } \\
\text { proches }\end{array}$ & $\begin{array}{c}\text { Extrême } \\
\text { gauche }\end{array}$ & $P C$ & $P S$ & Écologiste & UDF & RPR & $F N$ \\
\hline - Une fois & 64 & 47 & 19 & 46 & 43 & 25 & 52 \\
- Deux et trois fois & 30 & 31 & 37 & 37 & 38 & 35 & 35 \\
- Quatre fois et plus & 6 & 23 & 43 & 17 & 19 & 40 & 13 \\
\hline
\end{tabular}

Les jeunes qui témoignent d'une totale fidélité partisane sur l'ensemble de la période, soit onze années, sont de fait minoritaires : seuls $15 \%$ d'entre eux déclarent à six reprises se sentir proches d'un même parti. Les risques d'infidélité sont donc majeurs. En effet, plus de six jeunes sur dix (64\%) se sont déclarés, à un moment ou à un autre de leur parcours, proches d'un parti sans que ce même choix soit renouvelé. La mobilité est donc de mise. Du même coup, les chances pour les partis politiques de capter à un moment donné la sympathie d'un individu, et peut-être d'un électeur

1. La question de la proximité partisane n'a pas été posée à la vague 3 , et l'on dispose donc de cet indicateur pour les six autres vagues. 


\section{Anne Muxel}

potentiel, sont relativement élevées. Toutefois, cette éventualité ne se présente pas de la même façon pour tous, et ne fixe pas dans une même proportion des attaches durables. La fidélité escomptée par les partis ne s'établit pas avec la même intensité (tableau 7 ).

Le PS et le RPR suscitent la plus grande constance : $43 \%$ des jeunes déclarent se sentir proches du premier au moins quatre fois sur les six vagues d'enquête, et $40 \%$ du second. À droite, des écarts non négligeables différencient la fidélité envers le RPR et l'UDF. Contrairement au RPR, cette dernière fait l'objet d'un lien assez lâche. À gauche, la fidélité témoignée au PCF paraît relativement faible comparée aux autres partis traditionnels.

Les partis extrêmistes ne motivent pas des affinités très constantes. Seuls $6 \%$ des jeunes ont déclaré quatre fois et plus une proximité à un mouvement d'extrême gauche et seuls $13 \%$ au FN. L'un et l'autre provoquent des attirances sans doute plus sporadiques et circonstanciées : $64 \%$ des jeunes n'ont choisi qu'une seule fois l'extrême gauche et $52 \%$ le FN.

Enfin, la fidélité écologiste semble obéir à un modèle similaire et n'entraîne que peu de constance véritable.

\section{LA VOLATILITÉ DU VOTE}

Lorsque l'on examine la fidélité électorale, on retrouve une même logique de différenciation entre les grandes formations politiques classiques, représentant pour certaines d'entre elles un potentiel électoral important, d'une part, et les partis périphériques ou extrêmistes, d'autre part. Les premières suscitent des comportements plus constants tandis que les seconds font le jeu d'une grande volatilité.

Ayant reconstitué les trajets électoraux des jeunes à partir de leurs votes aux premiers tours de scrutin de quatre grandes élections, les deux présidentielles de 1988 et de 1995, correspondant respectivement aux vagues 2 et 6 de 1'enquête, et les deux législatives de 1993 et de 1997, correspondant aux vagues 5 et 7, la part de cette stabilité et de cette volatilité peut être appréciée ${ }^{1}$. La mesure de la fréquence des votes pour le même parti lors de ces quatre consultations fait apparaître une stabilité très différenciée (graphique 2).

On retrouve toujours une forte volatilité aux extrêmes. Les partis et mouvements d'extrême droite et surtout ceux d'extrême gauche ne semblent pas fixer d'attaches électorales durables, sur une période de près de dix ans : $87 \%$ des jeunes qui ont voté pour l'extrême gauche ne l'ont fait qu'une seule fois, et $61 \%$ de ceux qui se sont laissés tenter par le vote frontiste n'ont pas non plus renouvelé ce choix lors d'un autre scrutin.

Le vote écologiste apparaît lui aussi peu structuré et peu ancré dans des choix électoraux durables. Il présente les atours d'un vote de passage ou de circonstance, lié aux aléas et au contexte de l'offre électorale elle-même. Les trois-quarts des jeunes électeurs ( $73 \%$ ) ayant voté écologiste ne l'ont fait qu'une seule fois sur les quatre scrutins, et seule une minorité s'est montrée fidèle, $11 \%$, en répétant ce même choix trois ou quatre fois.

1. Sur la question de la mobilité électorale, on peut se reporter à l'article de Jean Chiche et de Jérôme Jaffré, «Mobilité, volatilité, perplexité », dans Daniel Boy, Nonna Mayer (dir.), L'électeur a ses raisons, op. cit. 
Graphique 2. Fidélité électorale

Fréquence du vote pour le même parti aux premiers tours des élections de 1988, 1993, 1995 et 1997

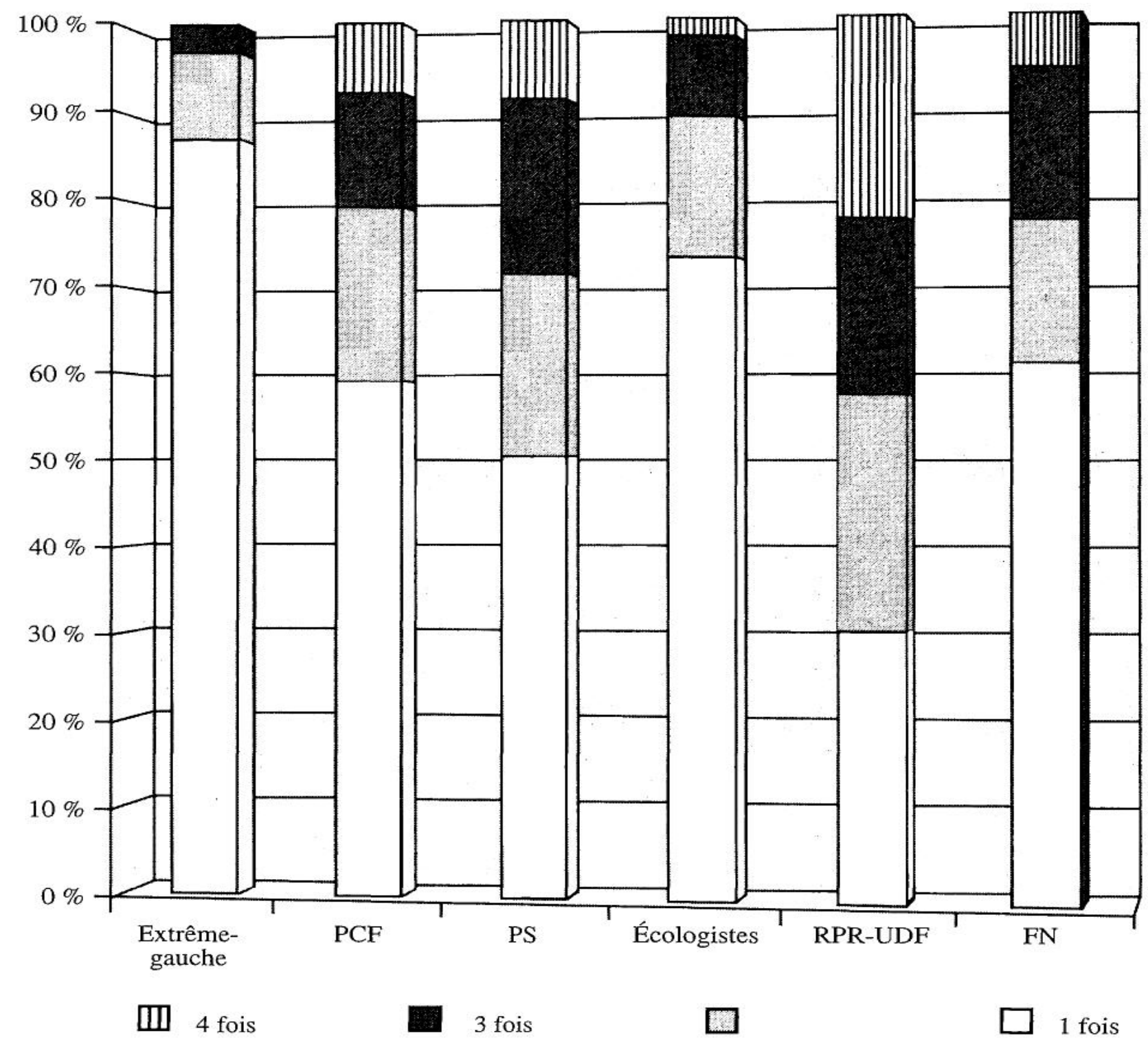

Les votes pour les partis traditionnels de gauche ou de droite sont nettement plus fidélisés, et tout particulièrement la coalition RPR-UDF : $43 \%$ des électeurs de cette dernière ont répété ce vote à trois ou quatre autres reprises, $29 \%$ des électeurs socialistes et $21 \%$ des électeurs communistes.

La reconstitution de l'ensemble de ces trajets de choix idéologiques, partisans ou électoraux, fait donc apparaître un jeu de positionnements au sein duquel la stabilité ne s'établit pas de façon indifférenciée ou équivalente. Qu'il s'agisse de l'échelle gauche-droite ou d'autres modalités d'activation des choix politiques, appréhendées au travers du sentiment de proximité partisane ou encore du vote, on observe une absence de structuration des positionnements extrêmes. En contrepoint, l'observation longitudinale fait apparaître de plus grandes chances de fidélité pour les positionnements plus modérés, centre-droit ou centre-gauche, ainsi que pour les partis politiques classiques ou fortement institutionnalisés. 


\section{Anne Muxel}

Mais progressons encore dans la compréhension du mouvement à l'œuvre dans la détermination et dans l'expression des choix politiques des jeunes. La mobilité connâ̂t-elle toujours la même amplitude, la constance les mêmes ressorts, quels que soient les indicateurs à partir desquels on appréhende ces choix ? En d'autres termes, le fait de se classer sur une échelle gauche-droite, de se déclarer proche ou pas d'un parti politique, ou encore de voter, contribue-t-il de façon équivalente à la structuration de l'identité politique ? Et notamment, connaît-il les mêmes chances de stabilité dans le temps?

\section{UNE STABILITÉ DIFFÉRENCIÉE DES INDICATEURS POLITIQUES}

J'ai effectué une mesure comparée de la stabilité dans le temps des trois indicateurs classiques du repérage politique que sont 1'autoclassement sur l'échelle gauchedroite, la proximité partisane et le vote, en prenant quatre moments d'observation, correspondant aux vagues $2,5,6$ et 7 de l'enquête. Quatre moments correspondant au calendrier électoral, au cours duquel alternent des élections présidentielles (1988, vague 2 , et 1995 , vague 6) et des élections législatives (1993, vague 5 et 1997 , vague 7 ) (tableau 8 ).

Tableau 8. Comparaison dans le temps de trois indicateurs classiques du repérage politique

Stabilité dans le temps de l'autoclassement sur l'échelle gauche-droite en sept positions

\begin{tabular}{ccccc}
\hline & $G D$ 88 & GD 93 & GD 95 & GD 97 \\
\hline GD 88 & - & .47 & .41 & .42 \\
GD 93 & - & - & .49 & .44 \\
GD 95 & - & - & - & .50 \\
GD 97 & - & - & - & - \\
\hline
\end{tabular}

Stabilité dans le temps de la proximité partisane

\begin{tabular}{lcccc}
\hline & PROXP 88 & PROXP 93 & PROXP 95 & PROXP 97 \\
\hline PROXP 88 & - & .38 & .35 & .37 \\
PROXP 93 & - & & .52 & .45 \\
PROXP 95 & - & - & - & .52 \\
PROXP 97 & - & - & - & - \\
\hline
\end{tabular}

Stabilité dans le temps du vote (1 $1^{\text {er }}$ tour $1988,1^{\text {er }}$ tour $1993,1^{\text {er }}$ tour $1995,1^{\text {er }}$ tour 1997$)$

\begin{tabular}{ccccc}
\hline & VOTE 88 & VOTE 93 & VOTE 95 & VOTE 97 \\
\hline VOTE 88 & - & .34 & .34 & .28 \\
VOTE 93 & - & - & .37 & .35 \\
VOTE 95 & - & - & - & .40 \\
VOTE 97 & - & - & - & - \\
\hline
\end{tabular}

Le tableau se lit ainsi : entre le positionnement des individus sur l'échelle gauche-droite en 1988 (vague 2) et leur positionnement en 1993 (vague 4), le taux de stabilité mesuré par le V de Cramer atteint le score de 47. La stabilité entre les positionnements est d'autant plus forte que l'on se rapproche de 1. 
La mesure du V de Cramer pour chacun de ces indicateurs fait apparaître une stabilité, - ou une mobilité -, des positionnements au fil du temps assez fortement différenciée. C'est l'autoclassement sur l'échelle gauche-droite qui s'affirme comme le plus stable. Même s'il n'échappe pas à une certaine érosion au fil du temps, il enregistre les coefficients les plus élevés (.42 entre les vagues 2 et 7). La proximité partisane s'avère un peu plus fragile, et la reconduction d'une préférence pour un parti politique entre la vague 2 et la vague 7 est moins assurée (.37). Mais c'est le vote qui est le plus volatile. Même si les votes lors d'élections rapprochées sont davantage corrélés, les coefficients sont dans l'ensemble plus faibles, et le $\mathrm{V}$ de Cramer entre les vagues 2 et 7 , mesurant un taux de probabilité de reproduire un même vote sur une longue période, soit neuf années, n'est plus que de. 28 .

Ainsi cette stabilité différenciée laisse-t-elle envisager des modes de perception de l'espace politique et de ses enjeux très différents, et ce, d'autant plus qu'ils sont reliés à des expériences sociales et politiques contrastées. L'examen de l'effet de certaines variables socio-démographiques et du niveau d'intérêt pour la politique sur cette stabilité vient encore complexifier cette hiérarchisation des trois registres d'implication politique qui ont été retenus, tout en en précisant le sens et la portée (tableau 9).

Tableau 9. Stabilité comparée des positionnements politiques selon différents critères socio-démographiques et selon l'intérêt pour la politique

\begin{tabular}{lccc}
\hline & $\begin{array}{c}\text { Autoclassement } \\
\text { sur l'échelle } \\
\text { gauche-droite } \\
\text { GD 88/GD 97 }\end{array}$ & Proximité partisane & Vote \\
\hline Études ++ & .54 & .47 & \\
Études - & .40 & .37 & .32 \\
Père cadre supérieur & .50 & .46 & .31 \\
Père ouvrier & .39 & .38 & .46 \\
Garçons & .47 & .38 & .31 \\
Filles & .37 & .35 & .32 \\
Intérêt politique & .44 & .41 & .26 \\
Non-intérêt politique & .46 & .33 & .33 \\
Ensemble & .42 & .37 & .27 \\
\hline
\end{tabular}

Le tableau se lit ainsi : entre le vote en 1988 (vague 2) des individus ayant poursuivi un cycle long d'études supérieures et leur vote en 1997 (vague 7), le taux de stabilité de leur vote mesuré par le V de Cramer s'établit à . 32 ; celui des individus n'ayant pas poursuivi d'études supérieures s'établit à .31.

Quel que soit l'indicateur pris en considération, l'origine sociale se révèle toujours la plus discriminante, une origine sociale modeste renforçant assez nettement les chances d'instabilité. Le niveau d'études entraîne le même type de fluctuation, excepté pour le vote dont la volatilité toujours plus grande semble échapper à ce type de détermination sociologique. 


\section{Anne Muxel}

On notera la différenciation assez marquée entre les filles et les garçons, ces derniers se montrant dans l'ensemble toujours plus stables dans leurs positionnements, quels que soient les indicateurs utilisés. Les filles seraient-elles plus malléables ou plus ouvertes au changement que les garçons?

L'intérêt pour la politique n'entraîne aucun écart s'agissant de l'autoclassement sur l'échelle gauche-droite, ce qui pourrait être interprété comme une preuve supplémentaire de l'existence du noyau dur représenté par le clivage gauche-droite, socle fondateur de l'identité politique mais ne requérant pas nécessairement d'investissement personnel ou de sollicitation particulière. En revanche, ceux qui ne s'intéressent pas à la politique ont beaucoup plus de chances de se montrer instables quant aux choix qu'ils vont effectuer au sein de l'offre politique partisane et électorale, cette dernière étant de loin la plus soumise aux aléas de l'arbitrage des choix.

La reconstitution de l'ensemble de ces trajets - gauche-droite, électoraux et partisans -, fait apparaître des formes de lien à la politique différenciées. C'est l'autoclassement sur l'échelle gauche-droite qui est le plus stable, la proximité partisane s'avère plus fragile, mais c'est le vote qui s'affirme comme le plus volatile.

Ces contrastes laissent supposer la coexistence de plusieurs grilles de lecture pour appréhender l'univers politique, se traduisant par des choix, et donc aussi par des actes, ne relevant pas nécessairement des mêmes mécanismes d'arbitrage ni non plus d'un même registre d'intentionnalité.

Les résultats présentés dans cet article révèlent deux dynamiques à l'œuvre dans le mouvement d'intériorisation et d'expression des positionnements politiques. Ils font apparaître, d'une part, une permanence assez consistante de l'orientation gauche-droite, - et vraisemblablement les attributs et les implications idéologiques que celle-ci présuppose -, qui s'impose comme un noyau dur et fondateur de l'identité politique, et, d'autre part, une relative malléabilité, une certaine fragilité, des choix dès lors que ceux-ci se portent sur des partis, des candidats et a fortiori sur des enjeux électoraux.

L'existence d'une stabilité différenciée des positionnements politiques selon les registres d'implication politique renvoie au travail de hiérarchisation et d'appropriation de la réalité politique au travers duquel s'effectue la socialisation politique, et plus concrètement la structuration de l'identité politique des individus. Le positionnement idéologique, mesuré par l'autoclassement sur l'échelle gauche-droite, est toujours le plus structurant en France, et correspond à une sorte de repérage élémentaire au sein de l'espace politique, élémentaire mais décisif dans la constitution des schèmes identitaires comme des argumentaires. Dans le processus de la socialisation politique ce premier repérage, ce premier décryptage de l'environnement politique, est sans doute celui qui s'est formé le plus tôt et qui enregistre les meilleures chances de durabilité. Mais bien que fortement structurant, ce noyau identitaire fondateur ne se traduit pas par des choix politiques concrets nécessairement conformes ou cohérents. La proximité partisane ou le vote apparaissent plus périphériques et plus incertains dans la structuration de l'identité politique. Mais ils n'en sont pas moins réels.

\section{L'HISTORICITÉ DES CHOIX}

Des travaux récents ont cherché à mettre en évidence les effets de génération et les effets de période sur les attitudes et sur les comportements politiques, mais aussi à les 


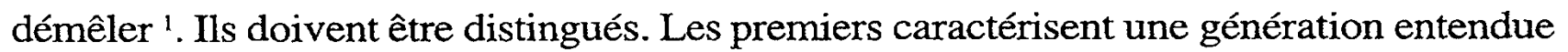
au sens d'un groupe ayant grandi et vivant dans une conjoncture historique donnée, y partageant l'expérience des mêmes événements au travers des singularités propres aux positions sociales de chacun; les seconds peuvent toucher l'ensemble des classes d'âge, et révéler, par exemple, des changements structurels profonds dans les comportements ou dans les mentalités. Les effets liés à la période sont souvent difficiles à analyser et à isoler car, bien que touchant toutes les classes d'âge, ils peuvent avoir des incidences différentes sur certaines d'entre elles, et donc se confondre avec les effets de génération.

Bien que difficiles à démêler, on peut néanmoins supposer que cette affectation différenciée de la période sur les générations interfère sur l'ensemble du processus de socialisation politique et cristallise plus ou moins durablement des attitudes et des comportements relevant d'une rencontre entre l'expérience individuelle et l'expérience historique collective.

\section{MESURER L'EMPREINTE D'UN ÉVÉNEMENT}

Grâce à la technique du panel, j'ai pu mesurer l'empreinte à plus ou moins long terme, tant sur le vote que sur d'autres dispositions à l'égard de la politique, d'un événement qui eut à l'époque un certain retentissement sur la scène politique française : le mouvement lycéen et étudiant de 1986. Il s'agissait d'un mouvement de protestation contre un projet de réforme de l'ensemble du système scolaire et universitaire, et notamment des procédures de sélection à l'entrée des universités, proposée par Alain Devaquet, alors ministre de l'Éducation nationale du gouvernement de Jacques Chirac. La première vague de l'enquête a démarré juste avant le début du mouvement, ce qui m'a permis, par le hasard de la rencontre entre l'actualité politique et le calendrier d'une observation scientifique, d'étudier les effets de la participation à une action politique concrète, et, dans le cas présent, de la participation à une mobilisation collective, sur les modes d'entrée en politique. La protestation serait-elle un moyen d'intégration politique?

Les événements de novembre-décembre 1986, en confrontant brutalement et directement les lycéens et les étudiants à la lutte politique, ont-ils marqué les choix politiques des jeunes de cette génération ? Par exemple, lors de l'élection qui vit les jeunes du panel voter pour la première fois, pour la présidentielle de 1988 ? Leur participation au mouvement a-t-elle influencé leur parcours politique ultérieur?

Un certain nombre d'indices, relevés dans les mois qui ont suivi le mouvement, révèlent que le mouvement eut des conséquences à l'échelle nationale sur les attitudes et sur les comportements politiques des jeunes. En 1988, on enregistre une plus forte proportion de jeunes inscrits sur les listes électorales qu'en 1986. Les taux d'inscription de ceux et de celles qui ont atteint 19,20 et 21 ans ont augmenté respectivement de 4,6 , de 2,1 , et de 5,5 points par rapport aux taux d'inscription des jeunes du même

1. Pour le cas français, on peut se reporter à l'ouvrage de Vincent Drouin, Enquête sur les générations et la politique (1958-1995), Paris, L'Harmattan, 1995. Des travaux relativement récents aux États-Unis se sont attachés à la mesure de ces effets différenciés, notamment Richard G. Braungart, Margaret Braungart, «Citizenship and Civic Education in the US in the 1990 s », dans Orit Ichilov (ed.), Citizenship and Citizenship Education in a Changing World: International perspectives, New York, Teachers' College, Columbia University, 1996 ou encore David Sears, N. A. Valentino, «Politics Matters : Political Events as Catalysts for Preadult Socialization », American Political Science Review, 91, 1997. 


\section{Anne Muxel}

âge observés en mars $1986^{\prime}$. Cette progression fut peut-être une réponse au mot d'ordre lancé à l'époque par la coordination lycéenne et étudiante, invitant les jeunes à s'inscrire ; elle révèle à coup sûr l'effet d'une motivation politique. Par ailleurs, la participation des jeunes à l'élection présidentielle de 1988 a été importante : $93 \%$ des 18-20 ans, $86 \%$ des 21-24 ans inscrits sur les listes électorales ont pris part au scrutin, soit un taux de participation assez semblable à celui que l'on a observé dans l'ensemble du corps électoral $(91 \%)^{2}$.

Au sein même du panel, plus de la moitié des jeunes de l'échantillon disent avoir participé, de façon plus ou moins active ou engagée, au mouvement : $26 \%$ ont participé à toutes ou à presque toutes les manifestations, $27 \%$ à quelques-unes, soit un total de $54 \%$ de participants, d'un côté, et $46 \%$ de non-participants, de l'autre ${ }^{3}$. Trois constats permettent d'expliciter l'influence de cette expérimentation de l'action collective :

- l'intérêt porté à la politique lors de la deuxième vague d'enquête est d'autant plus fort que la participation aux événements était élevée (78\% des jeunes ayant participé à toutes les manifestations disent s'intéresser à la politique, $70 \%$ lorsqu'ils ont participé à presque toutes, $66 \%$ à quelques-unes et $65 \%$ à aucune d'entre elles) ;

- il existe une relation entre la participation au mouvement et la participation à l'élection présidentielle de 1988:82\% des jeunes ont pris part au scrutin ; parmi les non-participants on ne compte que de rares abstentionnistes (4\%), mais beaucoup de non-inscrits puisque ceux-ci représentent encore à 20-21 ans $16 \%$. Les participants actifs au mouvement lycéen et étudiant ont davantage voté (87\% de participation parmi ceux qui ont été de toutes les manifestations contre $81 \%$ parmi ceux n'ayant participe à aucune d'entre elles) ;

- enfin, une participation active au mouvement de 1986 a entraîné une sanction des candidats de droite, et, plus particulièrement, un rejet de la candidature de Chirac. Lors du premier tour de scrutin, les participants à toutes ou à presque toutes les manifestations ont voté dans une large majorité pour un candidat de gauche, respectivement $68 \%$ et $62 \%$; lorsque la participation a été épisodique, les scores s'équilibrent avec un avantage pour la droite (39\% de votes à gauche et $46 \%$ de votes à droite) ; en revanche, les non-participants sont très nettement plus favorables à la droite $(67 \%$ de votes à droite contre seulement $23 \%$ de votes à gauche). Lors du second tour, les scores respectifs des deux candidats apparaissent comme étroitement liés à l'engagement ou non dans le mouvement : $82 \%$ de votes pour Mitterrand (contre $13 \%$ de votes pour Chirac) parmi les manifestants les plus actifs, $62 \%$ de votes pour Chirac (contre $29 \%$ de votes pour Mitterrand) parmi les non-participants. Ajoutons qu'au second tour, contrairement au premier, même dans le cas d'une participation minimale et épisodique au mouvement, les choix se portent davantage sur Mitterrand que sur Chirac ( $54 \%$ contre $40 \%$ pour ce dernier).

Les acteurs du mouvement apparaissent plus fortement ancrés à gauche : lors de la première vague d'enquête, c'est-à-dire au moment même des événements, $60 \%$ des jeunes ayant participé à toutes les manifestations et $49 \%$ à presque toutes se classent sur les positions de gauche (à droite, on n'en dénombre respectivement que $7 \%$ et $11 \%)$. La comparaison des classements sur l'échelle gauche-droite d'une année sur l'autre, fait apparaître que la participation au mouvement a entraîné une radicalisation

1. INSEE, Premiers résultats, 124, avril 1988.

2. Enquête post-électorale du CEVIPOF, 1988.

3. Le texte de la question posée lors de la deuxième vague d'enquête était: «Avez-vous participé aux grèves et aux manifestations du mouvement lycéen et étudiant, l'an dernier, en novembre et décembre 1986 ? à toutes les manifestations, à presque toutes, à quelques-unes, à aucune? ». 
à gauche supplémentaire. Si l'on observe chez les participants comme chez les non-participants au mouvement une augmentation des classements à gauche - révélateur de l'effet Mitterrand -, celle-ci est nettement plus marquée dans le cas d'une participation active au mouvement (on passe de $60 \%$ à $75 \%$ de classements à gauche, soit 15 points d'écart chez les jeunes ayant pris part à toutes les manifestations, contre $17 \%$ à $26 \%$, soit 9 points d'écart, parmi ceux qui n'ont participé à aucune d'entre elles).

Si la participation à une expérience politique collective engendre des conséquences durables, en tout cas à moyen terme, sur les orientations politiques et sur les comportements politiques des individus, en retrouve-t-on les traces à plus long terme? Quelle est l'intensité de l'imprégnation de cette expérience?

\section{UNE CRISTALLISATION POLITIQUE DE L’EXPÉRIENCE COLLECTIVE}

Ayant recueilli, lors de la vague 7, soit onze ans plus tard, les opinions des jeunes du panel sur le mouvement de novembre-décembre 1995, le mouvement social en France le plus important depuis Mai 68, protestant contre les réformes lancées par le gouvernement d'Alain Juppé concernant le service public et le système de protection sociale et de retraite, $\mathrm{j}$ 'ai pu vérifier non seulement la durabilité de cette empreinte, mais aussi constater qu'elle s'articule de façon différenciée avec l'orientation idéologique des individus, appréciée en termes de positionnement gauche, droite ou ni gauche ni droite ${ }^{1}$ (tableau 10 ).

Tableau 10. Influence de la participation au mouvement lycéens/étudiants de 1986 sur la solidarité envers le mouvement de novembre-décembre 1995

Solidaires du mouvement novembre-décembre 1995

Sont de gauche et ont participé à toutes les manifestations de $1986 \quad 75$

Sont de gauche et ont participé à quelques manifestations de 1986

Sont de gauche et n'ont participé à aucune manifestation de $1986 \quad 80$

Sont de droite et ont participé à toutes les manifestations de $1986 \quad 31$

Sont de droite et ont participé à quelques manifestations de $1986 \quad 14$

Sont de droite et n'ont participé à aucune manifestation de $1986 \quad 8$

Ne sont ni de gauche ni de droite et ont participé à toutes les manifestations de $1986 \quad 52$

Ne sont ni de gauche ni de droite et ont participé à quelques manifestations de $1986 \quad 47$

$\mathrm{Ne}$ sont ni de gauche ni de droite et n'ont participé à aucune manifestation de $1986 \quad 39$

Le tableau se lit ainsi : sur 100 jeunes qui sont de gauche et qui ont participé à toutes les manifestations de 1986, $75 \%$ sont solidaires du mouvement de novembre-décembre 1995.

La mobilisation collective définit un univers de pratiques et de représentations qui appartient à une culture politique de gauche. Cela est une nouvelle fois confirmé ${ }^{2}$. Les

1. La question était ainsi formulée: «À propos du mouvement de grève de novembredécembre 1995, vous vous êtes senti très solidaire, assez solidaire, peu solidaire, pas solidaire du tout?».

2. On peut se reporter à l'ouvrage collectif devenu maintenant un classique de la sociologie de l'action collective dirigé par Samuel H. Barnes et Max Kaase, Political Action. Mass Participation in Five Western Democracies, Beverly Hills, Londres, Sage Publications, 1979 ou encore en ce qui concerne le cas français au chapitre de Daniel Boy et Nonna Mayer « La participation politique », dans le livre collectif qu'ils ont dirigé, L'électeur a ses raisons, Paris, Presses de Sciences Po, 1997. 


\section{Anne Muxel}

manifestants du mouvement lycéen-étudiant de 1986 étaient de gauche et cette orientation s'était même renforcée dans les mois qui suivirent, on l'a vu. Onze ans plus tard, le lien à la gauche reste toujours dominant et une solidarité de fait avec l'action collective semble de fait toujours effective. Néanmoins les traces de leur participation au mouvement de 1986 ne semblent pas rentrer en considération pour expliquer leurs attitudes présentes. La solidarité ressentie envers le mouvement de novembredécembre 1995 connaît la même intensité, qu'ils aient ou non participé aux manifestations de l'époque. Le critère déterminant est idéologique, c'est l'orientation à gauche qui définit leur soutien potentiel.

Parmi les jeunes de droite comme parmi les indéterminés, ne se classant ni à gauche ni à droite, les effets de leur participation au mouvement de 1986, soit onze ans plus tôt, sont en revanche plus efficients. Bien que toujours plus réticents à l'égard du mouvement de 1995 que les jeunes de gauche, le fait d'avoir participé aux manifestations de 1986, accroît leurs chances de solidarité. L'expérience de l'action collective a pu façonner, dans une relative indépendance à l'égard des choix assignés par les positionnements idéologiques, des attitudes spécifiques, et, dans le cas présent, une plus grande ouverture, voire une solidarité possible, envers un autre mouvement de mobilisation et de protestation que celui qu'ils ont pu directement expérimenter.

La conjoncture historique et politique a donc un rôle actif dans la construction de l'identité politique. Elle peut agir sur les nombreuses dispositions antérieures produites au cours de la socialisation initiale ou sur les attributs de l'identité politique façonnés par les conditions d'insertion de l'individu dans la société. Ses effets sont le résultat d'une négociation que fait celui-ci, à partir de sa situation présente et de l'ensemble de ces dispositions, avec un événement donné. Elle offre ainsi de multiples occasions de rencontre en direct avec la politique. Le parcours politique de l'individu porte les traces de ces couches successives d'expériences, de rendez-vous manqués, de rencontres décisives et de moments de cristallisation.

L'existence d'une stabilité différenciée des positionnements politiques selon les registres d'implication politique renvoie au travail de hiérarchisation et d'appropriation de la réalité politique au travers duquel s'effectue la socialisation politique, et, plus concrètement, la structuration de l'identité politique des individus.

L'approche longitudinale fait apparaître le jeu d'activation et de désactivation des repères mobilisables par les individus dans une situation de choix. Elle permet de constater une adéquation flottante entre la structuration idéologique d'un individu et le processus des choix qu'il peut engager. Ainsi le vote entretient-il une relation de relative autonomie, et donc connaît-il une certaine volatilité, par rapport au mode de repérage constitué par le clivage gauche-droite, incontestablement plus stable dans le temps. Cette adéquation flottante ouvre une marge de mancuvre à la recomposition des choix individuels et introduit notamment un espace d'autonomie par rapport à des acquisitions ayant pu se cristalliser dans le cours de la socialisation primaire. C'est à l'intérieur de cet espace que peut s'exprimer l'idée d'acteur en politique, développant le sentiment d'agir en politique. Mais c'est aussi en cet endroit que peuvent être signifiés, sinon les signes de rupture ou d'anomie en politique, en tout cas les signes d'une crise de la représentation politique. L'individu peut s'orienter entre la gauche et la droite, et, pour autant, ne pas pouvoir se reconnaître au sein de l'offre politique qui lui est proposée. La palette de ses choix possibles connaît une latitude importante, allant 
du non-choix aux choix rationnels, c'est-à-dire les plus déconnectés de toute structuration idéologique et de toute prédisposition sociologique.

La participation à une expérience politique collective peut façonner des attitudes spécifiques, dans une relative indépendance à l'égard des choix assignés par les positionnements idéologiques, et modifier à terme les comportements politiques ultérieurs des individus. Il existe un lien entre la participation au mouvement lycéen-étudiant de 1986 et l'intensité de la solidarité ressentie à l'égard du mouvement de novembredécembre 1995. Une expérience de participation politique dans le temps de la jeunesse peut cristalliser certaines orientations politiques ayant des effets d'imprégnation durables sur les choix ultérieurs de l'individu.

Ainsi le temps continu de l'accumulation des apprentissages et des repères, structuré par le clivage gauche-droite, s'articule-t-il au temps discontinu des actes et des choix concrets, pour former la dynamique temporelle dans laquelle se déroule le processus de la socialisation politique dans le temps de la jeunesse.

Anne Muxel est sociologue au CNRS, chercheur au CEVIPOF (Centre d'étude de la vie politique française). Elle est 1'auteur de Individu et mémoire familiale, Paris, Nathan, 1996 (coll. «Essais et recherches ») et Les jeunes et la politique, Paris, Hachette, 1996. Elle vient de publier L'expérience politique des jeunes, Paris, Presses de Sciences Po, 2001. Ses travaux dans le champ de la sociologie politique $s$ 'attachent à la compréhension des formes du lien des individus à la politique, au travers de l'analyse de leurs attitudes et de leurs comportements (nouvelles formes d'expression politique, analyse du comportement électoral et des formes de recomposition des représentations comme des pratiques politiques) (CEVIPOF-Maison des sciences de l'homme, 54 Bd Raspail, 75006 Paris <muxel@msh-paris.fr>).

\section{RÉSUMÉ/ABSTRACT}

LES CHOLX POLITIQUES DES JEUNES À L'ÉPREUVE DU TEMPS. UNE ENQUÊTE LONGITUDINALE

Les années de jeunesse sont un temps de négociation et d'expérimentation, donc une période privilégiée pour observer le mouvement de formation et d'expression des choix politiques des individus. Seules les études longitudinales et l'observation de panel sur une longue durée permettent d'identifier le travail propre du temps sur la construction des choix et des comportements politiques et d'en apprécier la durabilité. Cet article présente des résultats tirés d'une enquête longitudinale menée auprès d'une cohorte de jeunes français âgés au départ de 18 ans, interrogés lors de toutes les grandes élections nationales, pendant près de douze ans c'est-àdire jusqu'à ce qu'ils atteignent l'âge de 30 ans. Sept vagues d'interrogations successives (1986, 1988, 1989, 1992, 1993, 1995,et 1997) ont été conduites. Les résultats de l'étude révèlent une structuration à la fois persistante et stable du clivage gauche-droite et corrélativement une plus grande fragilité du lien partisan ainsi qu'une volatilité encore plus marquée des choix électoraux. Ils montrent l'empreinte de la participation à une expérience politique collective sur les attitudes et les comportements politiques ultérieurs.

\section{THE STABILITY OF [FRENCH] YOUTH POLITICAL CHOICES. A LONGITUDINAL SURVEY}

Youth is an intensive period of transaction during which individuals, in different ways, enter adulthood and discover politics. Longitudinal studies allow us to observe not only the different ways of constructing one's "political self», but also the stability or instability of political 


\section{Anne Muxel}

choices during this relevant period. Some results from a longitudinal survey conducted for twelve years, between 1986 and 1998, on young people aged 18 in 1986, are presented. The cohort was questioned seven times, following the electoral agenda in France during that period (1986, 1988, 1989, 1992, 1993, 1995 and 1997). The data show significant variations in the stability of political choices throughout the period, depending on whether the measure refers to left-right self-placement, partisan identification or voting. Left-right identification is still the most structured and stable dimension in France, more so than partisan identification, and obviously much more than voting choice. The data also indicate the lasting impact of personal participation in collective action on later political attitudes and behavior. 